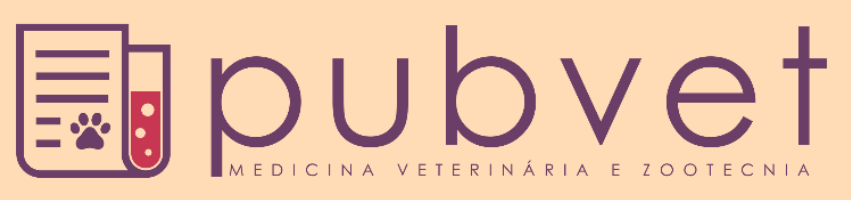

https://doi.org/10.31533/pubvet.v15n2a752.1-7

\title{
Eficiência dos sistemas de cultivo de camarões marinhos
}

\author{
Marcos Vinícius de Castro Freire ${ }^{1 *} \mathbb{C D}^{\bullet}$, Roosevelt de Araújo Sales Júnior ${ }^{2} \bullet$, Maria Gabriela \\ ${\text { Alves } \operatorname{Costa}^{30} \text {, Rosane Lopes Ferreira }}^{40}$
}

${ }^{I}$ Mestrando em Ciência Animal - Universidade Federal Rural do Semiárido - UFERSA, Mossoró - RN.

${ }^{2}$ Mestre em Ciência Animal - Universidade Federal Rural do Semiárido - UFERSA, Mossoró - RN.

${ }^{3}$ Mestre em Produção Animal - Universidade Federal Rural do Semiárido - UFERSA, Mossoró - RN.

${ }^{4}$ Doutoranda em Zootecnia - Universidade Estadual do Oeste do Paraná - UNIOESTE, Marechal Cândido Rondon - PR.

*Autor para correspondências, E-mail: marcos7phn@hotmail.com

Resumo. Um dos grandes desafios da carcinicultura é a maximização do cultivo diante das enfermidades. Vêm-se buscando práticas que auxiliem na expansão do setor e na sobrevivência dos animais nas distintas fases. No sistema multifásico, os camarões são estocados em altas densidades, em berçários intensivos primários e secundários, estratégia que tenta fortalecer as pós-larvas para a fase de engorda. $\mathrm{O}$ trabalho tem como por objetivos: (1) fazer um levantamento do histórico de produção de camarão no Brasil; (2) avaliar os benefícios do sistema multifásico na carcinicultura; (3) apresentar as vantagens, limitações, aplicações e viabilidade para o produtor dos sistemas de cultivo; (4) fazer um comparativo da eficácia dos sistemas de produção de camarão. A base de dados utilizada no estudo foi, artigos selecionados na base do portal de Periódicos da CAPES/MEC, no Google Acadêmico e no Scielo, e da produção de uma fazenda de camarão da região Nordeste do país, onde os valores de produção foram comparados em relação à sobrevivência final do cultivo. Foram avaliados os tratamentos, T1: Cultivo em viveiros escavados; T2: Povoamento em berçário primário, transferência para berçário secundário; T3: Povoamento em berçário primário, transferência para berçário secundário, transferência para viveiros escavados. A sobrevivência obtida, foi: T1: 98\%, T2: 45\%, T3: $32 \%$. Diante dos dados é possível identificar que a tecnificação dos sistemas necessita de profissionais capacitados, sistemas trifásicos apresentam grandes densidades, aumentando o contato com os animais durante as fases iniciais, além de apresentam um alto custo de produção, que dificultam o retorno financeiro ao produtor.

Palavras-chave: Carcinicultura, sistema intensivo, sustentabilidade

\section{Efficiency of marine shrimp farming systems}

Abstract. One of the great problems of shrimp culture is the maximization of crops and with it the emergence of new diseases, in the face of this problem, the aquaculture professionals, are seeking new practices that help in the expansion of the sector and the survival of the animals. In shrimp farming, animals can be submitted to different production systems comprising the various stages, such as single-phase, biphase or multiphase. In the multiphase system, the shrimp are initially stored in high densities, in primary intensive nurseries (BIP) and later in secondary nurseries (BIS), a strategy that strengthens the postlarvae and juveniles for the grow out or final phase. The goal of this work was to compare the effectiveness of shrimp production by evaluating the benefits of the multiphase system in shrimp culture and to discuss its possible advantages, limitations, applications and viability for the small farmer, as well as to the data used in the study came from the shrimp production in the Brazilian Northeast, where the production values of the single-phase system of grow out (direct settlement); and the multiphase system (primary nursery, 
secondary nursery, grow out) were compared in relation to the final survival of the shrimp. The following treatments were evaluated: Treatment 1 - (T1): Direct settlement in the ponds. Treatment 2 - (T2): Settlement in BIP, transfer to BIS; Treatment 3 - (T3): Settlement in BIP, transfer to BIS, transfer to ponds. The results of survival were: T1: $98 \%$, $\mathrm{T} 2: 45 \%, \mathrm{~T} 3: 32 \%$. In view of the data, it is possible to identify that the technification of the systems requires more skilled professionals, since three-phase systems present high densities, as well as increases the manipulation of the animals during the initial phases, in addition, these systems present a high cost of production, which hinder the small producer's financial return.

Keywords: Shrimp culture, intensive system, sustainability

\section{Eficiencia de los sistemas de cultivo de camarón marino}

Resumen. Uno de los grandes desafíos de la producción de camarón es la maximización del cultivo frente a las enfermedades. Se han estado buscando prácticas que ayuden en la expansión del sector y en la supervivencia de los animales en las diferentes fases. En el sistema multifásico, los camarones se siembran en altas densidades, en viveros intensivos primarios y secundarios, estrategia que busca fortalecer las post-larvas para la fase de engorde. Los objetivos de este documento son: (1) estudiar la historia de la producción de camarón en Brasil; (2) evaluar los beneficios del sistema multifásico en el cultivo de camarón; (3) presentar las ventajas, limitaciones, aplicaciones y factibilidad para el productor de los sistemas de cultivo; (4) hacer una comparación de la efectividad de los sistemas de producción de camarón. La base de datos utilizada en el estudio fueron los artículos seleccionados en la base del portal de Revistas CAPES / MEC, en Google Scholar y Scielo, y sobre la producción de una finca camaronera en el Noreste del país, donde se compararon los valores de producción en relación con la supervivencia final del cultivo. Se evaluaron los tratamientos, T1: Cultivo en viveros excavados; T2: Asentamiento en vivero primario, traslado a vivero secundario; T3: Asentamiento en vivero primario, traslado a vivero secundario, traslado a viveros excavados. La supervivencia obtenida fue: T1: $98 \%$, T2: 45\%, T3: 32\%. Frente a los datos, es posible identificar que la tecnificación de los sistemas requiere profesionales capacitados, los sistemas trifásicos tienen altas densidades, aumentando el contacto con los animales durante las fases iniciales, además de tener un alto costo de producción, lo que dificulta el retorno económico al productor.

Palabras clave: Cultivo de camarón, sistema intensivo, sostenibilidad

\section{Introdução}

A carcinicultura é uma atividade econômica que tem apresentado grande crescimento em termos mundiais nos últimos anos (Cavalheiro, 2015), atividade agroindustrial que segundo a FAO (2018) teve produção mundial de 7,9 milhões de toneladas, produzidos em 2016. É uma atividade com potencial de suprir a elevada demanda alimentar, utilizando novas tecnologias e investimentos no setor, trazendo estabilidade aos sistemas de produção.

Apesar do crescimento, o setor passou por dificuldades com a mancha branca, enfermidade causada por um vírus que se manifesta no desenvolvimento inicial, calcificando e mudando a cor do crustáceo, que causa mortalidade em massa dos camarões (Everton, 2020). Segundo a ABCC (2016), a coexistência com tal enfermidade, visa a busca por alternativas, procedimentos, métodos e práticas de cultivo, que melhorem a produtividade e minimizem os prejuízos, e diminua o uso dos recursos naturais.

Pensando na coexistência, o setor voltou seu olhar para o cultivo em sistemas extensivos em viveiros escavados, e intensivos em estufas, com princípios de biossegurança. A região Nordeste do Brasil é líder em produção no país, com mais de $99 \%$ da produção nacional (Rodrigues \& Borba, 2013). A criação do camarão pode ocorrer em diferentes fases de cultivo, podendo ser monofásica, bifásica e trifásica (ABCC, 2016). Barbosa (2018) ressaltam que o uso de estufas produz pós-larvas de camarão mais uniformes, saudáveis, diminui o tempo de cultivo, o contato dos animais com o meio externo e melhora a sobrevivência. 
Os objetivos deste trabalho foram (1) fazer um levantamento do histórico de produção de camarão no Brasil; (2) avaliar os benefícios do sistema multifásico na carcinicultura; (3) apresentar as vantagens, limitações, aplicações e viabilidade para o produtor dos sistemas de cultivo; (4) fazer um comparativo da eficácia dos sistemas de produção de camarão.

\section{Material e métodos}

Base de dados de produção no Brasil de 2004 a 2016

A base de dados utilizada no estudo foi proveniente de artigos selecionados na base do portal de Periódicos da CAPES/MEC, no Google Acadêmico e no Scielo, e também dados, disponibilizados pela FAO, com auxílio do software Fishstat J, para o levantamento do histórico de produção de camarão no Brasil. Fazendo-se um recorte temporal, no intervalo dos anos de 2004 a 2016.

\section{Base de dados experimentais}

A base de dados utilizada na avaliação experimental foi proveniente da produção de uma fazenda de cultivo de camarão L. vannamei, da região Nordeste do Brasil, cidade de Mossoró - RN. Com um período de coleta de dados de julho a setembro de 2017.

Segundo estudos de Persyn \& Aungst (2001), Albertim-Santos et al. (2014), Melo (2018) e Fróes et al. (2007), a utilização de sistemas multifásicos em cultivos aquáticos com finalidade de aumento de produção, teve origem na década de 70 , intensificando-se na década de 90 , utilizando-os como estágios de transição entre a larvicultura e o crescimento final.

Os valores de produção no estudo, do sistema monofásico dos viveiros de engorda com povoamento direto; e do sistema multifásico, contendo berçário intensivo primário (BIP), berçário intensivo secundário (BIS) e viveiro de engorda, foram comparados em relação à sobrevivência final do cultivo, avaliando a sobrevivência dos animais em função da tecnologia aplicada.

As densidades de estocagem empregadas foram consideradas elevadas, com alimentação, temperatura e parâmetros físico-químicos da água controlados e acompanhados constantemente durante todo o ciclo de cultivo.

\section{Tratamentos utilizados}

Foram avaliados os seguintes tratamentos:

Tratamento 1 - (T1): Povoamento direto das pós-larvas nos viveiros escavados.

$>$ Tratamento 2 - (T2): Povoamento das pós-larvas em berçários intensivos primários (BIP), e posterior transferência para berçários intensivos secundários (BIS);

Tratamento 3 - (T3): Povoamento das pós-larvas em berçários intensivos primários (BIP), com transferência para berçários intensivos secundários (BIS), e posterior transferência para viveiros escavados.

\section{Descrição dos berçários}

Para Krummenauer et al. (2012), a utilização de estufas, oportuniza a criação de camarões peneídeos em regiões com clima subtropical e temperado, sendo alvo de pesquisas visando melhores condições aos animais e melhor desempenho zootécnico, como utilização de princípios de biossegurança. $\mathrm{O}$ berçário é uma estrutura em forma normalmente retangular, num sistema de estufa, utilizado como estratégia de convivência com enfermidades. No estudo, eles possuíam as dimensões de 0,2 ha (100 m x $20 \mathrm{~m}$ ) com $300 \mathrm{~m}^{3}$ para o BIP, e BIS com $1.700 \mathrm{~m}^{3}$. Feita de aço galvanizado e recoberto em cima e nas laterais, por lona leitosa de alta resistência inclusive na parte inferior, foi utilizado o solo da própria fazenda como substrato acima da lona, possuindo portas nas partes da frente, de trás e na lateral junto com a janela lateral. Os berçários foram divididos em duas áreas, ou seja, BIP e BIS.

\section{Dados limnológicos dos berçários}

Os berçários possuíam tratamento térmico da água controlado e constante em torno de $32^{\circ} \mathrm{C}$. A aeração foi suprida por um soprador de 10 c.v. de potência, e com oito aeradores, ambos aeradores do 
tipo paddle de dois c.v. de potência, uniformemente bem distribuídos por toda estrutura. Para garantir a segurança do sistema de cultivo, a estrutura ainda possuía um grupo gerador ligado em casos de emergência, assim como em horários de pico, como alternativa de baratear o consumo de energia.

A temperatura da água foi mantida em torno de $32^{\circ} \mathrm{C}$ e foi monitorada durante todo o dia e noite com auxílio de um termômetro digital, fixado em cada uma das fases, com sensor que permanecia submerso na água. O termômetro exibia os dados de temperatura dentro e fora d'água. Os parâmetros de oxigênio dissolvido $(\mathrm{OD})$ e temperatura $\left(\mathrm{T}^{\circ} \mathrm{C}\right)$, foram aferidos diariamente às 16:00 h, as 20:30 $\mathrm{h}$, as 00:00 $\mathrm{h}$, as 03:30 h, as 07:00 h, por um parametrista.

\section{Manejo dos berçários}

O manejo dos berçários foi realizado por técnicos, que se revezavam entre si para que houvesse o suporte $24 \mathrm{~h}$. Possuía ao lado, uma estrutura construída para apoio, visando o armazenamento dos insumos e equipamentos utilizados durante o cultivo.

\section{Viveiros escavados}

Os viveiros escavados com dimensões de cinco ha, com alturas médias de 1,4 e 2,0 m. Possuíam formatos retangulares, com platô (parte central dos viveiros) e valas (maior concentração de animais). As estruturas possuíam comportas de drenagem e abastecimento em formato de ' $\mathrm{Y}$ ', sendo sempre em direções opostas. As comportas possuíam jogos de telas de 1.000 micras no abastecimento, que eram trocadas de acordo com o tamanho do camarão.

\section{Resultados e discussão}

O recorte temporal, filtrado entre os anos de 2004 a 2016, mostra que apesar das adversidades o setor conseguiu se manter, e tende a ser uma atividade importante para a economia do país. Na figura 1 observa-se os declínios na produção de camarão marinho (nos últimos anos) se deram em função da síndrome da mancha branca, requerendo pensamentos e estratégias para lidar com a enfermidade.

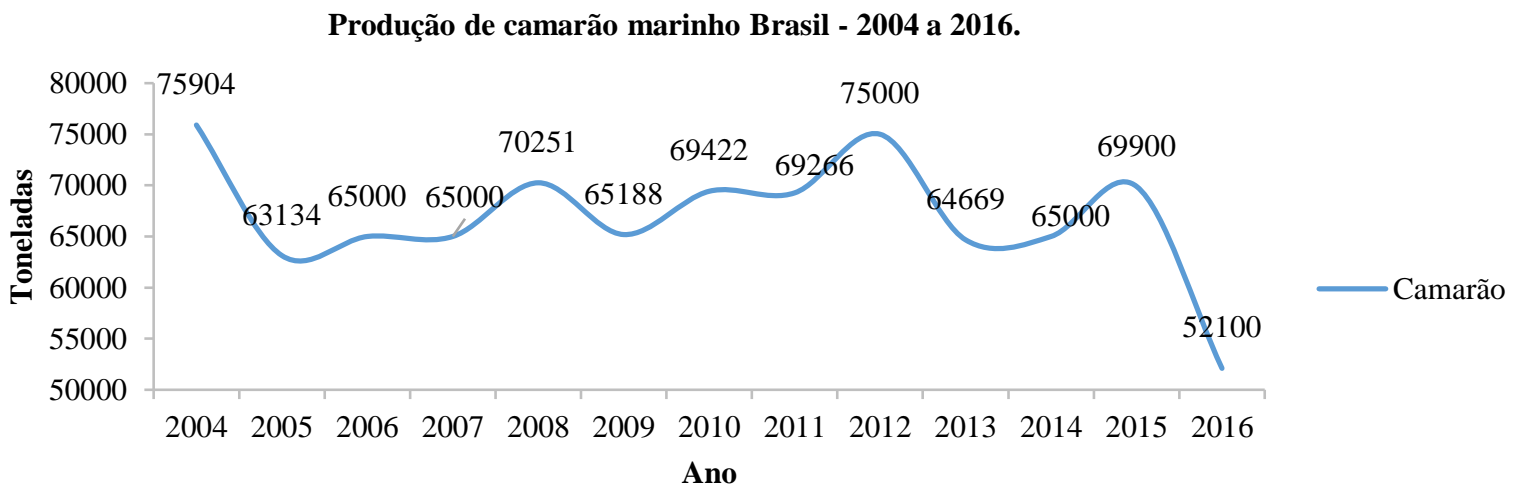

Figura 1. Evolução da produção de camarão marinho no Brasil de 2004 a 2016. Fonte: Fishstat J (2017).

Apesar dos controles ambientais e limnológicos favorecerem a produção, reduzindo o tempo do ciclo cultivo, e em alguns casos melhorar a sobrevivência e os índices zootécnicos dos camarões cultivados, o fato do vírus da mancha branca ter chegado ao Nordeste do país e dizimado grande parte da produção, fez-se necessário voltar o olhar para o uso de novas tecnologias.

Com a expansão da aquicultura, diversos sistemas de cultivo surgiram (Melo, 2018), obtendo valores produtivos maiores. O que na década de 1980 era baseado apenas em sistemas extensivos, com grandes áreas de superfície e baixa densidade de animais (Wasielesky et al., 2006) expandiu numa diversidade de sistemas de cultivo. No estudo experimental, os resultados dos dados produtivos de sobrevivência dos camarões, durante o período de avaliação do ciclo, expressos na tabela 1 , demonstram que, em função da complexidade das práticas, houve prejuízos à sobrevivência dos organismos de cultivo e consequentemente prejuízos financeiros. 
Apesar da adoção de tecnologias garantirem o sucesso da carcinicultura no mundo, com fatores fundamentados em tecnologias importadas e aprimoradas, as quais passaram por uma intensa experimentação e validação e tecnologia (Abrunhosa, 2011), o uso no Brasil deste pacote tecnológico ainda requer adaptações, melhorias e avanços. Segundo (Kubitza, 2009), o constante monitoramento é mais complexo de se realizar, exigindo intervenções e ajustes de acordo com o monitoramento.

Tabela 1. Dados produtivos de sobrevivência de camarões $L$. vannamei.

\begin{tabular}{llllll}
\hline Tratamentos & Descrição & Povoamento (pls) & Peso (g) & Sobrevivência (\%) & Tempo (dias) \\
\hline T1 & VE & 500.000 & 7 & 98 & 38 \\
T2 & BIP>BIS & 400.000 & 7 & 45 & 88 \\
T3 & BIP>BIS >V.E & 246.000 & 4,3 & 32 & 40 \\
\hline
\end{tabular}

VE: viveiro escavado; BIP: berçário intensivo primário; BIS: berçário intensivo secundário.

Apesar das estratégias de estufas virem dando certo mundialmente, com inúmeras vantagens como mostra a tabela 2, ainda não foram assertivas no Nordeste brasileiro, e ainda requerem aperfeiçoamento e melhorias.

Tabela 2. Vantagens do uso de estufas

Vantagens do uso de estufas mundialmente

Eficiência Econômica Do Emprego E Retorno Do Capital

Utilização De Mão-De-Obra Especializada, Aumentando O Número De Empregos

Conservação Do Meio Ambiente, Em Função Das Melhorias Na Qualidade De Água

Controle Amplo E Melhor Desenvolvimento, Manejo E Cuidado Com Os Animais

Fonte: Tropical Estufas (2019).

No Brasil, sistemas de cultivo intensivo podem viabilizar a produção de camarões como atividade sazonal em áreas de grandes flutuações térmicas e/ou halinas, fazendo com que as pesquisas sobre o manejo desses cultivos se tornem indispensáveis (Maia et al., 2012), por apresentar alguns obstáculos que dificultam a execução e desempenho produtivo.

Os principais entraves, apresentados na maioria das fazendas de cultivo no Nordeste brasileiro giram em função da mão de obra humana. A tabela 3 apresenta os principais benefícios e os entraves da atividade.

Tabela 3. Principais benefícios e entraves do uso de sistemas intensivos (estufas).

\begin{tabular}{|c|c|}
\hline Benefícios & Autores \\
\hline Melhor aclimatação & ABCC (2016), Maia et al. (2012) \\
\hline Maior controle da qualidade de água & ABCC (2016), Avnimelech(2006) e Krummenauer et al.(2012) \\
\hline Pós-larvas maiores e mais resistentes & $\underline{\mathrm{ABCC}(2016)}$, Avnimelech (2006) \\
\hline Diminuição do tempo de engorda & ABCC (2016), Monteiro (2005) \\
\hline Local de estocagem prévia & ABCC (2016), Kubitza (2009) \\
\hline Melhor manejo alimentar & Lopes et al. (2012) \\
\hline Ciclo com proteção das estufas & $\mathrm{ABCC}(2016)$ \\
\hline Entraves & $\overline{\text { Autores }}$ \\
\hline Aumento do manuseio dos animais & $\mathrm{ABCC}(2016)$ \\
\hline Menos tolerante a erros de manejo & $\underline{\mathrm{ABCC}(2016)}$ \\
\hline Requer técnicos treinados e capacitados & $\overline{\mathrm{ABCC}(2016)}$, Kubitza (2009) \\
\hline Custo de construção mais alto & ABCC (2016), Monteiro (2005) \\
\hline Custo de manejo mais alto & ABCC (2016), Kubitza (2009) \\
\hline
\end{tabular}

\section{Conclusões}

Analisando os dados de produção de camarão marinho no Brasil de 2004 a 2016, foi possível fazer um levantamento para avaliar o comportamento da produção da carcinicultura marinha, com intuito de servir de respaldo à comunidade acadêmica. Percebeu-se que é possível que a atividade se consolide e seja uma grande fonte proteica, sendo necessário que haja adaptações para melhorias.

É possível identificar que a tecnificação dos sistemas necessita de profissionais mais capacitados, uma vez que sistemas multifásicos apresentam grandes densidades, assim como aumenta a manipulação 
dos animais durante as fases iniciais, além disso, esses sistemas apresentam um alto custo de produção, que dificultam o retorno financeiro ao pequeno produtor. A mancha branca dificultou um pouco esse balanço, e apesar de estar inativada em altas temperaturas qualquer descuido pode colocar em risco todo o sistema.

Apesar dos benefícios do sistema de berçário intensivo como, melhores condições de adaptação das pós-larvas às condições físico-químicas da água, o maior controle da qualidade da água e da nutrição das pós-larvas (incluindo uma apropriada oxigenação, em que o fornecimento de pós-larvas maiores e mais resistentes garantam um crescimento satisfatório), melhor desempenho do camarão e menor tempo de cultivo na etapa seguinte, devido a falhas, o uso de berçários ainda não é viável para o pequeno produtor, frente aos desafios do sistema, por requerer processos mais criteriosos e menos tolerantes a erros, requerendo técnicos bem treinados, sem falar no custo da construção e de engorda que é mais alto do que o do sistema simples.

Sendo assim ainda se faz necessário, estudos mais profundos a respeito da viabilidade das estufas para pequenos produtores, assim como, mais investimentos em qualificação e técnicas no setor.

\section{Referências}

ABCC. 2016 - Associação Brasileira de Criadores de Camarão. 2016. Programa de qualificação especial em boas práticas de manejo e biossegurança para micro, pequenos produtores de camarão. ABCC. Natal - RN, Brasil.

Abrunhosa, F. A. (2011). Curso técnico em pesca e aquicultura: carcinicultura. In Governo FederalMinistério da Educação. Instituto Federal de Educação, Ciências e Tecnologia do Pará-IFPA. Universidade Federal do Rio Grande do Norte.

Albertim-Santos, C. J., Santos, D. L., \& Mendes, P. (2014). Uso de modelos matemáticos para avaliação das variáveis de manejo do Litopenaeus vannamei (Boone, 1931)/Use of mathematical models for evolution of the management variables Litopenaeus vannamei (Boone, 1931). Acta of Fisheries and Aquatic Resources, 2(2), 28-39. https://doi.org/10.2312/Actafish.2014.2.2.28-39

Avnimelech, Y. (2006). Bio-filters: the need for an new comprehensive approach. Aquacultural Engineering, 34(3), 172-178. https://doi.org/10.1016/j.aquaeng.2005.04.001

Barbosa, M. M. N. (2018). Berçário e engorda do camarão marinho Litopenaeus vannamei (Boone, 1931) na fazenda Camar Aquamaris, João Pessoa-PB. Brasil.

Cavalheiro, T. B. (2015). Avaliação do efluente da dessalinização utilizado no cultivo de camarão marinho litopenaeus vannamei. Universidade Federal de Campina Grande.

Everton, F. A. (2020). Influência do sistema de cultivo no desempenho zootécnico e resposta à infecção pelo Vírus da Síndrome da Mancha Branca em pós-larvas de camarão marinho Litopenaeus vannamei (Boone, 1931). Universidade Federal Rural de Pernambuco.

FAO. (2018). The State of Food Security and Nutrition in the World 2018 - Building climate resilience for food security and nutrition.

Fróes, C. N., Abe, M. P., Wasielesky Junior, W. F. B., Hernandez, C. P., \& Cavalli, R. O. (2007). Efeitos de dietas práticas com diferentes níveis de proteína bruta na sobrevivência e crescimento do camarãorosa Farfantepenaeus paulensis (Pérez-Farfante, 1967). Atlantica, Rio Grande, 29(1), 25-34. DOI: 10.5088/atlântica.v29i1.741

Krummenauer, D., Seifert Junior, C. A., Poersch, L. H. da S., Foes, G. K., Lara, G. R. de, \& Wasielesky Junior, W. (2012). Cultivo de camarões marinhos em sistema de bioflocos: análise da reutilização da água. Atlântica, Rio Grande, 34(2), 103-111. doi: 10.5088/atl.2012.34.2.103

Kubitza, F. (2009). Manejo na produção de peixes. Panorama Da Aquicultura, 19(14), 14-23.

Lopes, D. L. de A., Suita, S. M., Bueno, C., Wasielesky Júnior, W. F. B., \& Poersch, L. H. S. (2012). Determinação da densidade de estocagem ótima do camarão rosa Farfantepenaeus brasiliensis produzindo em tecnologia de bioflocos durante a fase de berçário. Atlântica, Rio Grande, 34(2), 113120. DOI: 10.5088/atl.2012.34.2.113

Maia, E. P., Modesto, G. A., Brito, L. O., \& Gálvez, A. O. (2012). Crescimento, sobrevivência e produção de Litopenaeus vannamei cultivado em sistema intensivo. Pesquisa Agropecuária 
Pernambucana, 17(1), 15-19. https://doi.org/10.4322/pap.2012.004

Melo, J. M. da C. (2018). Cultivo do camarão marinho Litopenaeus vannamei em sistema intensivo e semi-intensivo na Fazenda Aquarium Aquicultura do Brasil Ltda. Brasil.

Monteiro, O. L., Gumes, F. L. L. 2005. Piscicultura Super-intensiva como Proposta para o Desenvolvimento Sustentável de uma Pequena Comunidade Rural. 2005. XII SIMPEP - Bauru, São Paulo.

Persyn, H., \& Aungst, R. (2001). Global shrimp OP: 2001-Preliminary Report-Nursery. Global Aquaculture Advocate, 4(4), 34-35.

Rodrigues, J., \& Borba, M. (2013). Carcinicultura brasileira: estatísticas e revelações. Revista Feed \& Food, 72.

TROPICAL ESTUFAS. 2019. Carcinicultura - Estufas / Viveiro para Camarão. 2019. Bragança Paulista - SP. Disponível em:https://tropicalestufas.com.br/carnicicultura-estufas-para-camarao/ Acesso em: 20 de abril de 2019.

Wasielesky, W., Atwood, H., Stokes, A., \& Browdy, C. L. (2006). Effect of natural production in a zero exchange suspended microbial floc based super-intensive culture system for white shrimp Litopenaeus vannamei. $\quad$ Aquaculture, 258(1), 396-403. https://doi.org/10.1016/j.aquaculture.2006.04.030

\section{Histórico:}

Recebido: 15 de setembro de 2020

Aprovado: 23 de outubro de 2020.

Disponível online: 22 de dezembro de 2020.
Licenciamento: Este artigo é publicado na modalidade Acesso Aberto sob a licença Creative Commons Atribuição 4.0 (CC-BY 4.0), a qual permite uso irrestrito, distribuição, reprodução em qualquer meio, desde que o autor e a fonte sejam devidamente creditados. 